Document downloaded from:

http://hdl.handle.net/10251/67078

This paper must be cited as:

Carsí Rosique, M.; Sanchis Sánchez, MJ.; Díaz Calleja, R.; Riande, E.; Nugent, MJD. (2013). Effect of slight crosslinking on the mechanical relaxation behavior of poly(2ethoxyethyl methacrylate) chains. European Polymer Journal. 49(6):1495-1502. doi:10.1016/j.eurpolymj.2012.12.012.

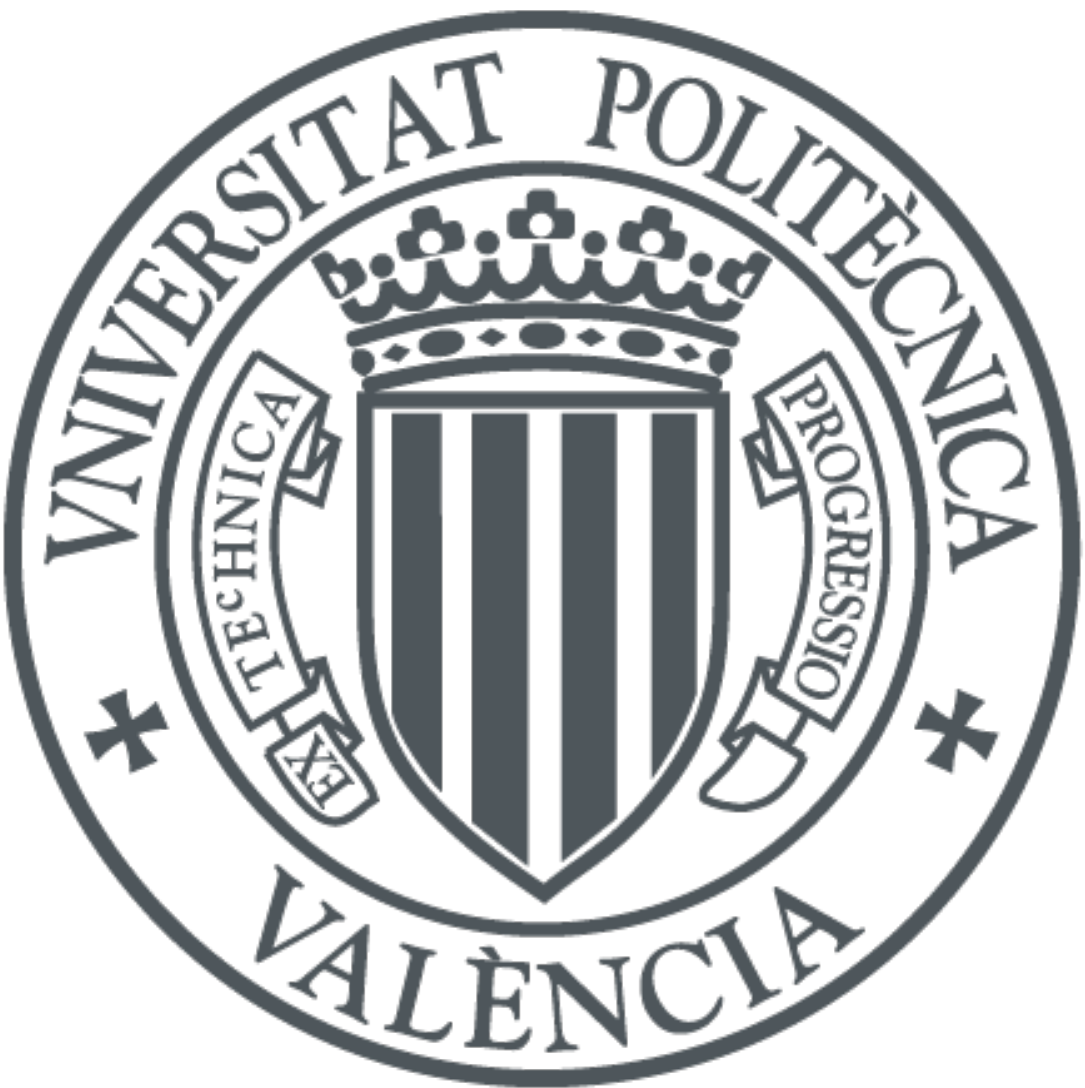

The final publication is available at

http://dx.doi.org/10.1016/j.eurpolymj.2012.12.012

Copyright Elsevier

Additional Information 


\title{
Effect of slight crosslinking on the mechanical relaxation behavior of poly (2-ethoxyethyl methacrylate) chains
}

\author{
M. Carsí ${ }^{\text {a,* }}$, M. J. Sanchis ${ }^{\text {a }}$, R. Díaz-Calleja ${ }^{\text {a }}$ \\ ${ }^{a}$ Energy Technological Institute (ITE), Universitat Politècnica de València, Camino de Vera \\ s/n 46022, Valencia, Spain. \\ E. Riande ${ }^{b}$
}

${ }^{\mathrm{b}}$ Institute of Polymer Science and Technology (ICTP), Spanish National Research Council (CSIC), Juan de la Cierva 3, 28008, Madrid, Spain.

M.J.D. Nugent ${ }^{c}$

${ }^{\mathrm{c}}$ Athlone Institute of Technology, Dublin Rd, Athlone, Co. Westmeath, Ireland

\begin{abstract}
The synthesis, thermal and mechanical characterizations of uncrosslinked and lightly crosslinked poly(2-ethoxyethyl methacrylate) are reported. The uncrosslinked poly(2ethoxyethyl methacrylate) exhibits in the glassy state two relaxations called in increasing order of temperature, the gamma and beta processes respectively. These are followed by a prominent glass-rubber or alpha relaxation. By decreasing the chains mobility by a small amount of crosslinking, the beta relaxation disappears and the peak maximum associated with the alpha relaxation is shifted from $268 \mathrm{~K}$ to $278 \mathrm{~K}$, at $1 \mathrm{~Hz}$. An investigation of the storage relaxation modulus of the crosslinked polymer indicates two inflexion points that presumably are related to segmental motions of dangling chains of the crosslinked networks and to cooperative motions of the chains between crosslinking points. Nanodomains formed by sidegroups flanked by the backbone give rise to a Maxwell-Wagner-Sillars relaxation in the dielectric spectra that have no incidence in the mechanical relaxation spectra.
\end{abstract}




\section{Introduction}

Chains dynamics is a flourishing field of research in polymer science due in part to the practical applications of these studies in engineering [1]. Furthermore, the prediction of the mechanical performance of polymeric structures requires an understanding of the chain dynamics of polymers. As is well-known, the glass-rubber or $\alpha$-relaxation that arises from segmental motions of molecular chains, freezes at $T_{g}$. This characteristic, in conjunction with the fact that the $\alpha$ relaxation is the dominant process in chain dynamics until molecular chains disentanglement occurs, leads us to consider the glass-rubber relaxation as the precursor of the glassy state and the viscous flow. Moreover, the $\alpha$ relaxation also occurs in oligomers of low molecular weight, well below the entanglement condition [2-3]. Besides the glass rubber relaxation, the relaxation spectra of polymers in the frequency domain present secondary relaxations produced by conformational transitions of the chains backbone or motions of flexible side-groups [4-6]. Unlike the glass-rubber relaxation and the normal mode process [7] that reflects chains disentanglement, secondary relaxations remain operative below $\mathrm{T}_{\mathrm{g}}$.

Secondary relaxations can have a great impact on the mechanical properties of polymers in the glassy state [4-6, 8-9]. For example, chair-inverse-chair conformational transitions of cyclohexyl in poly(cyclohexyl methacrylate) produce an ostensible secondary $\beta$ relaxation that causes a significant decrease of the real relaxation modulus of the polymer in the glassy state [10-12]. Since to date, while no quantitative theory that describe the glass-rubber relaxation and the secondary relaxations in terms of the chemical structure has been formulated, $(i)$ the theory of the total dielectric relaxation strength for the $\alpha$-process is wellestablished in terms of molecular dipole moments [4-6, 13-22] and (ii) much success has been achieved in understanding the characteristic behavior of the dielectric $\alpha$ relaxation through computer "molecular dynamics" simulations [23-29]. In this sense, actually the design of polymers with specific physical properties relies on empirical rules based on experimental studies of the relaxation properties of polymers with different chemical structures.

Poly(n-alkyl methacrylate)s and poly(n-alkyl acrylate)s have been widely used in the study of chain dynamics owing to the great dependence of the properties of the members of the series on the length of the alkyl residue [4-9, 30-53]. The first member of the series, poly(methyl methacrylate) (PMMA), is widely used in household and automotive applications [54]. The fact that PMMA has a glass-transition temperature ca. $100 \mathrm{~K}$ above that of poly(methyl acrylate) (PMA) puts in evidence that hindering of the conformational transitions 
by effect of the methyl group rigidly attached to the polymer backbone. This results in the increase of the $\mathrm{T}_{\mathrm{g}}$ of PMMA [4]. Moreover, the tacticity of the PMMA has a significant influence in the dynamics of this polymer, thus the $\mathrm{T}_{\mathrm{g}}$ value of the isotactic form is lower than that corresponding to the syndiotatic form. The $\beta$ peak is located at somewhat lower temperatures for the isotatic polymer than for the sindiotactic polymer. Moreover, the magnitude of the $\beta$-relaxation is also very influenced by tacticity. Thus, whereas the height of the $\beta$ peak is about twice that of the $\alpha$ peak for the convetional polymer, the opposite situation exists in the case of the isotatic polymer [4].Moreover, the X-ray spectra of poly(n-alkyl methacrylate)s melts with $\mathrm{n} \geq 2$, which shows that these polymers are heterogeneous systems formed by nanodomains integrated by side-chain groups flanked by the chains backbone [37, 44-45]. Cooperative motions of the side chains in the domains produce an $\alpha_{E P}$ peak located at higher frequencies than the glass-rubber relaxation arising from segmental motion of the chains backbone.

Recently, the dielectric relaxation behavior of poly(2-etoxyethyl methacryate) (PEOEMA) was studied [55]. PEOEMA can schematically be obtained by replacing a methylene group of poly(pentyl methacrylate) for an ether group. This polymer has been used as drug-eluting stent coating for percutaneous coronary interventions, providing durable, robust coatings with precise control over rapamycin elution rates [56]. The formation of nanodomains visible in the X-ray spectra of poly(n-pentyl methacrylate)s is not detected in poly(2-ethoxyethyl methacrylate) (PEOEMA) [40-41, 45]. The X-ray pattern of this polymer only shows a single peak that reflects the average distance of side chains groups. However, the X-ray spectra of slightly crosslinked PEOEMA exhibit, in addition to the peak appearing in the uncrosslinked polymer, another transition presumably arising from correlations between the chains backbone [55]. The similarity of these spectra with those of poly(n-alkyl methacrylate)s suggests the presence of nanodomains in the crosslinked PEOEMA network. Hereinafter, the acronym of crosslinked polymer will be CEOEMA. In view of these antecedents, a mechanical-dynamical study was undertaken on PEOEMA and CEOEMA. The aim of the study was to determine whether the differences observed in the mesoscopic structure of the uncrosslinked and crosslinked polymers, which affect the dielectric spectra of these systems, will also affect their mechanical relaxation spectra.

\section{Experimental Section}


Commercial monomer 2-ethoxyethyl methacrylate (Aldrich, 99\%) (Figure 1) and the cross linker diethylene glycol dimethacrylate (Aldrich, 98\%) (Figure 2) were purified by distillation under high vacuum. Dioxane (Aldrich; 99\%) was distilled twice: the first time over sodium hydroxide and the second time over sodium. 2, 2'-Azobisisobutyronitrile (AIBN, Fluka; 98\%) was recrystallized from methanol and dried under high vacuum at room temperature. All other materials and solvents used for the synthesis were commercially available and they were used as received unless otherwise indicated.

Poly 2-ethoxyethyl methacrylate (PEOEMA) was obtained by radical polymerization of 2-ethoxyethyl methacrylate in dioxane solution using 2.5 wt $\%$ of 2,2'azobisisobutyronitrile (AIBN) as initiator. The reaction was carried out in nitrogen atmosphere, at $343 \mathrm{~K}$, for $5 \mathrm{~h}$. The polymer was precipitated with methanol, washed several times with this organic compound and finally dried under high vacuum at room temperature. The number and weight average molecular weights of PEOEMA were measured by size exclusion chromatography (SEC) in a Perkin-Elmer apparatus with an isocratic pump serial 200 connected to a differential refractometric detector (serial 200a). Two Resipore columns (Varian) were conditioned at $70{ }^{\circ} \mathrm{C}$ and used to elute the samples $(1.0 \mathrm{mg} / \mathrm{mL}$ concentration) at $0.3 \mathrm{~mL} / \mathrm{min}$ HPLC-grade $\mathrm{N}, \mathrm{N}^{\prime}$-dimethyl formamide (DMF) (Scharlau) supplemented with $0.1 \mathrm{v} / \mathrm{v} \% \mathrm{LiBr}$. Calibration of SEC was carried out with monodisperse standard poly(methyl methacrylate) samples in the range of $2.9 \times 10^{3}$ to $480 \times 10^{3}$ obtained from Polymer Laboratories. The values of $\mathrm{M}_{\mathrm{n}}$ and $\mathrm{M}_{\mathrm{w}}$ were 82600 and $250800 \mathrm{~g} \cdot \mathrm{mol}^{-1}$, respectively.

CEOEMA was prepared by radical copolymerization of 2-ethoxyethyl methacrylate and ethylene glycol dimethacrylate, the mass fraction of the latter comonomer or crosslinking agent in the feed being $1 \mathrm{wt} \%$. The polymerization reaction took place at $343 \mathrm{~K}$ in a silanizedglass mold of about $100 \mu \mathrm{m}$ thickness, in oxygen free atmosphere. A crosslinked film, without bubbles, was obtained which was washed with methanol and further dried at room temperature under vacuum.

In order to characterize the polymer, Fourier Transform Infrared Spectroscopy (FTIR) was used to study the structure and complexation of the polymers. Infrared spectroscopy was performed on a Nicolet Avator 360 FTIR spectrometer, with a 32 scan per sample cycle. For each sample, scans were recorded from 4000 to $400 \mathrm{~cm}^{-1}$ with a resolution of $4 \mathrm{~cm}^{-1}$. The spectra obtained show a signal at $1700 \mathrm{~cm}^{-1}$ associated with the $\mathrm{C}=\mathrm{O}$ stretching vibration of carboxylic group, one signal at $2900 \mathrm{~cm}^{-1}$ due to $\mathrm{CH}_{2}$ stretching and the signal at $1125 \mathrm{~cm}^{-1}$ associated with C-O-C asymmetric stretching. 
Differential scanning calorimetry (DSC) of PEOEMA and CEOEMA was carried out with a TA Instruments DSC Q-10 differential scanning calorimeter calibrated with indium. The measurements were carried out in the range of $193 \mathrm{~K}$ to $423 \mathrm{~K}$ at a heating rate of $10 \mathrm{~K} \cdot \mathrm{min}^{-1}$ under nitrogen atmosphere.

Dynamic mechanical measurements of PEOEMA and CEOEMA were performed by means of a Dynamic Mechanical Analyzer (TA Instruments DMA Q800) calibrated with steel standards. The measurements were carried out in the tension mode on molded probes of $10 \times 7 \times 0.1 \mathrm{~mm}$ over the temperature range from $133 \mathrm{~K}-400 \mathrm{~K}$. Before the measurements, samples were dried in vacuum oven at $303 \mathrm{~K}$ to remove moisture. Measurements were carried out at $1 \mathrm{~K} \cdot \mathrm{min}^{-1}$ heating rate, at frequencies of $0.3,1,3,10$ and $30 \mathrm{~Hz}$. In the case of CEOEMA, the measurements of the Young's modulus in the frequency domain were extended up to $100 \mathrm{~Hz}$.

\section{Results}

The DSC thermograms for PEOEMA and CEOEMA exhibit well developed endotherms associated with the glass transition temperature. The values of $\mathrm{T}_{\mathrm{g}}$ of the samples, estimated as the temperature at the midpoint of the endotherms, and the heat capacity increments $\left(\Delta c_{p}\right)$ at $\mathrm{T}_{\mathrm{g}}$ were $278 \mathrm{~K}, 0.27 \mathrm{~J} / \mathrm{gK}$ and $268 \mathrm{~K}, 0.28 \mathrm{~J} / \mathrm{gK}$ for CEOEMA and PEOEMA respectively. Thus, the crosslinking agent reduces the number of chains thermally activated and the chain mobility and raises the $\mathrm{T}_{\mathrm{g}}$ ca $10 \mathrm{~K}$, and diminished the change in specific heat capacity $\left(\Delta c_{p}\right)$. This effect can be understood in terms of decreasing free volume.

Storage and loss moduli isochrones for PEOEMA and CEOEMA, over the temperature window 133-400K, are shown in Figures 3 and 4, respectively. The loss isochrones corresponding to PEOEMA show three differentiated relaxations zones. Around $270 \mathrm{~K}(1 \mathrm{~Hz})$, the dynamic mechanical response is dominated by the glass-rubber relaxation, but at lower temperatures, in the glassy state, a broad absorption centered around $210 \mathrm{~K}$ is evident. This absorption is labeled $\beta$ relaxation. Finally, the loss isochrones show the presence of a $\gamma$-relaxation process below $145 \mathrm{~K}$. The three relaxations observed in the isochrones of PEOEMA are reduced to two relaxations in the isochrones corresponding to CEOEMA. Thus the loss isochrones for the latter system exhibit at $1 \mathrm{~Hz}$ a sub-glass absorption centered at $155 \mathrm{~K}$ ( $\gamma$ relaxation) followed in increasing order of temperature by the glass-rubber relaxation ( $\alpha$ process) centered at $280 \mathrm{~K}$ at the same frequency. As would be expected, the location of the 
$\gamma$ peak is shifted to higher temperatures as frequency increases, and the intensity of the peak increases as the frequency of the isochrones increases. The location of the $\alpha$ relaxation is also displaced to higher temperatures as frequency increases, but the intensity of the relaxation seems to be independent on the frequency of the isochrone.

The more significant differences between the mechanical behavior of PEOEMA and CEOEMA are the following: (i) the $\gamma$ relaxation of former system is located at slightly lower temperature than that of the latter; (ii) the $\beta$ relaxation detected in the isochrones of PEOEMA disappears in CEOEMA, and (iii) as a consequence of the reduction in chains mobility caused by crosslinking the location of the $\alpha$ relaxation is shifted to higher temperature, in accordance with the DSC results.

\section{Discussion}

Since the $\gamma$-relaxation in the spectra falls just on the low temperature limit reached by the apparatus, it is difficult to estimate the parameters describing the relaxation. Using the Heijboer assumption that states that the Arrhenius equation describing the temperature dependence of the relaxation times associated with the secondary relaxations of most flexible polymers has the same pre-exponential factor $\tau_{0}=10^{-14.5} s$ [10], the activation energies of the $\gamma$ relaxations of PEOEMA and CEOEMA are, respectively, $44.4 \mathrm{~kJ} \cdot \mathrm{mol}^{-1}$ and 47.4 $\mathrm{kJ} \cdot \mathrm{mol}^{-1}$

An alternative method of obtaining directly the activation energies is to express the loss relaxation results in terms of compliance data, taking into account that the following inequalities: $T\left(E^{\prime \prime}{ }_{\max }\right)<T\left(\tan \delta_{\max }\right)<T\left(D^{\prime \prime}{ }_{\max }\right)$. This means that the loss compliance relaxations are shifted to higher temperatures than the loss modulus relaxation processes. As can be seen in Figure 5, the compliance $\gamma$ relaxation covers a temperature range that allows the estimation of the activation energy, strength and shape parameter of the process.

Sub-glass relaxations are usually nearly symmetric peaks, and therefore both isochrones and isotherms can be characterized by means of the Fuoss-Kirkwood equation [57]

$$
D^{\prime \prime}=D_{\max }^{\prime \prime} \cdot \operatorname{sech} m\left[\frac{E_{a}}{R}\left(\frac{1}{T}-\frac{1}{T_{\max }}\right)\right]
$$

where $T_{\max }$ is the temperature where $D^{\prime \prime}$ have a maximum value $\left(D_{\max }^{\prime \prime}\right), E_{\mathrm{a}}$ is the apparent activation energy, $R$ is the gas constant, and $m$ is an empirical parameter $(0<m<1)$ related to 
the broadness of the relaxation in the sense that the smaller $m$, the wider the distribution is. The value of $m=1$ corresponds to a single relaxation time (Debye peak). The strength of the mechanical relaxation peak can be calculated from the relationship $\Delta D=2 D^{\prime \prime}{ }_{\max } / m$ [4].

The parameters of eq (1) fitting the compliance $\gamma$ processes of PEOEMA and CEOEMA were determined from a multiple nonlinear regression analysis of the experimental results, varying the three characterizing peak parameters (i.e., $D^{\prime \prime}{ }_{\max }, m E_{\mathrm{a}} / R, T_{\max }$ ). In the inset of Figure 5, an example of the quality of the fit is shown. In the case of the PEOEMA the $\gamma$ absorption is followed by the $\beta$ process. However, the latter process is not well defined because the right side of the relaxation overlaps with the low temperature side of the $\alpha$ absorption. As a consequence, only the parameters that describe the $\gamma$ relaxation were estimated and their values are collected in Table I. The errors associated with the parameters show the quality of the fit at the frequencies investigated. The parameter $m_{\gamma}$ does not show a noticeable dependence on frequency. Alternatively, the low values of $m_{\gamma}$ are an indication of the distributed character of the $\gamma$ process and, as expected, the temperature dependence of the relaxation exhibits Arrhenius behaviour (ARRH). The activation energy calculated from the Arrhenius plot was $54 \mathrm{~kJ} \cdot \mathrm{mol}^{-1}$ and $55 \mathrm{~kJ} \cdot \mathrm{mol}^{-1}$ for PEOEMA and CEOEMA, respectively.

It is of interest to compare the activation energies of the secondary processes with those obtained from dielectric results. The dielectric loss isochrones for PEOEMA and CEOEMA, taken from previous work [55], are shown in Figure 6. Arrhenius plots for the dielectric $\gamma$ and $\beta$ relaxations of PEOEMA and for the $\gamma$ relaxation of CEOEMA are shown in Figure 7. From these plots, values of $29.9 \pm 0.4 \mathrm{~kJ} \cdot \mathrm{mol}^{-1}$ are obtained for the activation energies of the dielectric $\gamma$ relaxation of both PEOEMA and CEOEMA and $41.6 \pm 0.3 \mathrm{~kJ} \cdot \mathrm{mol}^{-1}$ for the activation energy of the dielectric $\beta$ relaxation of PEOEMA. The molecular origin of the secondary dielectric relaxation can be qualitatively explained as follows. The ester group of the side chains of PEOEMA and CEOEMA has a dipole moment of $1.78 \mathrm{D}$ that forms an angle of $153^{\circ}$ with the $\mathrm{C}\left(\mathrm{CH}_{3}\right)-\mathrm{C}(\mathrm{O})$ bond while the dipole moment of the ether group bisects the skeletal $\mathrm{CH}_{2}-\mathrm{O}-\mathrm{CH}_{2}$ bond and has value of $1.23 \mathrm{D}$ [18]. In all trans conformation both dipoles have nearly the same direction and therefore the polarity of the all trans conformation of the side groups reaches the maximum value. On the other hand the $\mathrm{C}(\mathrm{O})-\mathrm{O}$ bonds are restricted to the trans states and the $\mathrm{O}-\mathrm{CH}_{2}$ bonds strongly prefer the trans conformation. However, since the $\mathrm{CH}_{2}-\mathrm{CH}_{2}$ bonds prefer the gauche conformation, conformational transitions about these bonds produce dielectric activity which can be responsible for the $\gamma$ relaxation observable in the dielectric and mechanical spectra. It is more difficult to elucidate 
the origin of the $\beta$ relaxation appearing in the mechanical and dielectric spectra of PEOEMA. However, the fact that this relaxation is absent in the spectra of CEOEMA suggests that it proceeds mainly from motions in the polymer backbone which are impeded by crosslinking. The fact that the mechanical $\gamma$ relaxation exhibits an activation energy nearly $80 \%$ higher than the dielectric $\gamma$ process suggests that the molecular motions involved in the mechanical process are more complex than in the dielectric one. Combined molecular motions about $\mathrm{C}\left(\mathrm{CH}_{3}\right)-\mathrm{C}(\mathrm{O})$ and $\mathrm{CH}_{2}-\mathrm{CH}_{2}$ bonds of the side groups may be an origin of the mechanical $\gamma$ process. As for the mechanical $\beta$ relaxation, the absence of this process in the spectra of CEOEMA suggests that the crosslinking suppresses that process. Therefore the mechanical $\gamma$ relaxation of PEOEMA must be attributed to local cooperative motions of the backbone.

The temperature dependence of the mean relaxation time associated with the mechanical glass-rubber relaxation was analyzed in the context of the free volume theory by means of the Vogel-Fulcher-Tammann-Hesse (VFTH) equation [58-60]

$$
\ln f_{\text {max }}=A-\left[\frac{M}{T-T_{v}}\right]
$$

where $A$ and $M$ are constants, $T_{v}$ is an empirical parameter related to the Kauzmann temperature or the temperature at which the conformational entropy is zero and $f_{\max }$ is the frequency at which $E$ " passes through the maximum value. The parameters of eq.(2) that fit the Arrhenius plots are $A=(31.5 .0 \pm 4.2), M=(1514 \pm 140) \mathrm{K}, T_{v}=(233.1 \pm 7.2) \mathrm{K}$ for CEOEMA and $A=(23.3 \pm 0.2), M=(1114 \pm 10) \mathrm{K}, T_{v}=(218.3 \pm 2.2) \mathrm{K}$, for PEOEMA.

By comparing eq (2) with the Doolittle expression [61-62], the fraction of free volume at the glass transition temperature, $\phi_{g} / B$, and the free volume expansion coefficient $\alpha_{f}=(1 / V)(\partial V / \partial T)_{p}$ are estimated from the following expressions

$$
\begin{aligned}
& \frac{\phi_{g}}{B}=\frac{T_{g}-T_{v}}{M} \\
& \frac{\alpha_{f}}{B}=\frac{1}{M}
\end{aligned}
$$

According to the Cohen-Turbull theory, $B$ is a parameter close to the unit related to the ratio between the critical volume for a relaxation process to take place and the volume of the segments intervening in the process. Assuming $B=1$, the values of the relative free volume at $\mathrm{T}_{\mathrm{g}}$ for PEOEMA and CEOEMA were, respectively, $0.045 \pm 0.001$ and $0.030 \pm 0.009$, whereas the values of $\alpha_{\mathrm{f}}$ amount to $(0.90 \pm 0.01) \times 10^{-3} \mathrm{~K}^{-1}$ and $(0.66 \pm 0.19) \times 10^{-3} \mathrm{~K}^{-1}$. It is worth noting that the values of $\phi_{g}$ and $\alpha_{f}$ are nearly twice the values reported for this quantities for 
most flexible polymers, presumably as consequence of the fact that the relaxation curves only extend over a rather limited span of frequencies and temperatures [9]. In spite of that, the values of $\alpha_{f}$ are very close to the results found from the temperature dependence of the dielectric $\alpha$ relaxation, about $0.9 \times 10^{-3} \mathrm{~K}^{-1}$ for both systems, whereas the results estimated for $\phi_{\mathrm{g}}$ from the dielectric experiments are 0.040 and 0.037 for PEOEMA and CEOEMA, respectively.

A detailed inspection of the isochrones corresponding to the storage relaxation modulus of CEOEMA shows two inflexion points centered in the vicinities of $250 \mathrm{~K}$ and $280 \mathrm{~K}$, which apparently reflects the presence of two relaxations. This is confirmed by the curve representing the derivative of the real component of $E^{\prime}$ with respect to the temperature. The curve $d E^{\prime} / d T$ for CEOEMA, shown in Figure 8, exhibits two peaks in the vicinity of the calorimetric glass transition temperature, absent in the curve $d E^{\prime} / d T$ corresponding to PEOEMA. The low temperature peak, centered at $250 \mathrm{~K}$ cannot be attributed to the $\beta$ peak detected around $200 \mathrm{~K}$ in the relaxation loss spectra of PEOEMA. Although the glass transition temperature depends on the free volume and temperature, thermodynamical considerations have shown recently that the contribution of thermally activated conformational transitions to the glass-rubber relaxation is more important than the volume [8]. According to Fujimori and Oguni [63], the non-Arrhenius behavior of the $\alpha$ relaxation could be interpreted as caused by changes in the activation energy with temperature. The value of this parameter can be calculated as a function of temperature using the thermodynamic relationship

$$
\left[\frac{\partial \ln f}{\partial(1 / T))}\right]_{E^{\prime}}\left[\frac{\partial(1 / T))}{\partial E^{\prime}}\right]_{\ln f}\left(\frac{\partial E^{\prime}}{\partial \ln f}\right)_{T^{-1}}=-1
$$

Since the activation energy is given by the following equation

$$
E_{a}=-R\left(\frac{\partial \ln f}{\partial(1 / T)}\right)_{E^{\prime}}
$$

and taking into account the Schwarzl and Struik [64] approximation

$$
E^{\prime \prime} \cong \frac{\pi}{2} \frac{d E^{\prime}}{d \ln f}
$$

the following equations that relates the activation energy to the components of the complex modulus is obtained [65-68]

$$
E_{a}=\frac{\pi R T^{2}}{2 E^{\prime \prime}} \frac{d E^{\prime}}{d T}
$$


Curves depicting the variation of the activation energy for PEOEMA and CEOEMA in the whole temperature window, evaluated by using eq. (7), at $30 \mathrm{~Hz}$, are shown in Figure 8. Figure 8 also shows the temperature dependence, of the $E^{\prime}, E^{\prime \prime}$ and $\mathrm{dE}^{\prime} / \mathrm{dT}$ at the same frequency. Two well developed peaks are observed for CEOEMA centered at the same temperatures as the less defined $d E \% d T$ peaks whereas a single peak associated with $\mathrm{T}_{\mathrm{g}}$ appears in the distribution of activation energies of PEOEMA. Moreover, the temperature dependence of the apparent activation energy corresponding to the $\alpha$-relaxation was evaluated in terms of the VFTH parameters $\left(E_{a}^{\alpha}(T)=\left[R \cdot M /\left(1-\left(T_{v} / T\right)\right)^{2}\right]\right)$ [64]. As we can observe, according to the VFTH prediction, the $E_{a}$ decreases with the temperature increasing, and the values obtained near $\mathrm{T}_{\mathrm{g}}$ are similar to those one obtained by using eq. (7). In view of these results, the first peak, centered at $240 \mathrm{~K}$, corresponding to the distribution of activation energies in CEOEMA seems to be associated with a low temperature glass rubber relaxation, neither detected in the calorimetric thermograms nor in the dielectric relaxations, presumably arising from segmental motions of dangling chains in the chemically crosslinked network. The location of the network, nearly $15 \mathrm{~K}$ below the peak associated with the $\mathrm{T}_{\mathrm{g}}$ of PEOEMA, suggests that the dangling chains have relatively low molecular weight.

Previous studies showed important differences between the microstructure at mesoscopic level of PEOEMA and CEOEMA [55]. Thus the X-ray diffractogram patterns of the crosslinked polymer exhibit two peaks centered at $\mathrm{q}=5.6 \mathrm{~nm}^{-1}$ (peak I) and $\mathrm{q}=12.8 \mathrm{~nm}^{-1}$ (peak II). This pattern reflects the formation of nanodomains formed by the side groups flanked by the skeletal bonds in such a way that peak I arises from interaction between the skeletal bonds limiting the nanodomains whereas the peak at higher angles is produced by interactions between the side groups of the nanodomains. However, the X-ray diffractogram of PEOEMA only presents the second peak at high angles $\left(q=12.8 \mathrm{~nm}^{-1}\right)$, therefore ruling out the presence of nanodomains in the melt of this polymer. The differences in microstructure of CEOEMA and PEOEMA are reflected in the dielectric spectra of the respective systems at high temperatures, shown in Figure 9. The isochrones corresponding to the dielectric modulus of PEOEMA present two well defined peaks: the low temperature peak associated with the $\alpha$ relaxation is followed by a rather sharp peak centered at $313 \mathrm{~K}$ arising from interfacial polymer-electrode phenomena. However, the high temperature peak of CEOEMA presents in addition to the peak corresponding to the $\alpha$ relaxation an ostensible and wide peak that it is the result of two overlapping peaks (centered at 353 and 393K). The low temperature peak reflects the MWS relaxation arising from transport of charges in the bulk over a 
considerable distance with respect to the atomic or segments caused by the heterogeneity of the system [69-73]. It can be concluded that the nanodomains to which we alluded before are responsible for this relaxation. As in the case of PEOEMA, the deconvoluted high temperature peak is produced by interfacial polymer electrode phenomena. Owing to the crosslink nature of CEOEMA the loss modulus isochrones for these systems were extended to temperatures well-above $T_{\mathrm{g}}$. The corresponding isochrones plotted in parallel with the loss dielectric modulus in Figure 9 do not show an additional absorption above that of the $\alpha$ relaxation. However the nanodomains present in CEOEMA do not seem to have any incidence in the response of the system to mechanical perturbation forces.

\section{Conclusions}

Uncrosslinked PEOEMA chains exhibit two secondary relaxation processes in the glassy state which in increasing order of frequency are called $\gamma$ and $\beta$ relaxations. The $\beta$ relaxation is suppressed by slightly crosslinking the PEOEMA chains with only the $\gamma$ relaxation remaining. The $\gamma$ relaxation may be produced by conformational transitions about the $\mathrm{OCH}_{2}-\mathrm{CH}_{2} \mathrm{O}$ bonds of the alcoholic residue whereas the $\beta$ relaxation may arise from local motions of the polymer backbone.

The storage relaxation modulus isochrones of CEOEMA present two inflexion points in the glass-rubber transition, centered at the peak maxima of the variation of activation energy with temperature in the transition. These phenomena, neither detected in the calorimetric thermograms nor in the loss dielectric spectra, presumably are associated with segmental motions of the dangling chains of the networks (low temperature inflexion point) and the segmental motions of the chains between crosslinked points (high temperature inflexion).

The mesoscopic structure of the crosslinked polymer that gives rise to a MaxwellWagner-Sillars relaxation in the dielectric spectra at high temperature does not seem to have any incidence in the relaxation mechanical spectra.

\section{Acknowledgments}

We thank Dr. J. Guzman (Madrid) for providing us with the CEOEMA sample. This work was financially supported by the DGCYT and CAM through the Grant MAT2008-06725C03. 


\section{References}

[1] Graham N.B. Controlled drug delivery systems. Chemical Industry 482-486, 1990.

[2] Ezquerra TA, Zolotukhin M, Privalño VP, Baltá-Calleja FJ, Nequlqueo G, García C, de la Campa JG, de Abajo J. Journal of Chemical Physics 1999; 110(20): 10134.

[3] Roland CM, Casalini R, Paluch M, Chemical Physics Letters 2003; 367:259.

[4] McCrum, N.G.; Read, B.E.; Williams, G. Anelastic and Dielectric Effects n Polymeric Solids, Wiley, London, 1967.

[5] Kremer, F.; Schönhals, A. In Broadband Dielectric Spectroscopy, Springer: Berlin, 2003.

[6] Riande, E.; Díaz-Calleja, R., Electrical Properties of Polymers, Dekker: New York, 2004

[7] Stockmayer W. H. Pure Appl. Chem. 1967; 15:539.

[8] Mpoukouvalas K, Floudas G, Williams G. Macromolelcules 2009; 42:4690.

[9] Ferry JD, Viscoelastic Properties of polymers, $2^{\text {rd }}$ ed New York: John Wiley\&Sons, 1961. [10] Heijboer, J. Mechanical Properties of Glassy Polymers Containing Saturated Rings, Ph.D. Thesis, University of Leiden, 1972.

[11] Ribes-Creus A, Gómez-Ribelles JL, Díaz-Calleja R. Polymer 1995; 26(12): 1849.

[12] Domínguez-Espinosa G, Sanchis MJ, Díaz-Calleja R, Pagueguy C, Gargallo L, Radic D. Polymer 2005; 46: 8028 .

[13] Volkenstein M. V., Configurational Statistics of Polymer Chains. Interscience Publishers, Inc., New York, 1963.

[14] Glarums H, Journal of Chemical Physics, 1960; 33:371-5.

[15] Cole RH. Journal of Chemical Physics 1965;42:637-43

[16] Cole RH. Theories of dielectric polarization and relaxation. Progress in Dielectrics, vol 3, Heywood 1961.

[17] Cook M, Watts DC; Williams G., Trans. Faraday Soc., 1970; 66: 2503-2511

Williams G. Advances in Polymer Science 1979; 33 : 59-92

[18] Riande E, Saiz E. Dipole Moments and Birefringence of Polymers, Prentice Hall, Edgenwoodcliff, N.J. 1992.

[19] Sanchis MJ, Domínguez-Espinosa G, Díaz-Calleja R, Guzmán J, Riande E. The Journal of Chemical Physics 2008; 129:054903.

[20] Sanchis MJ, Carsí M, Ortiz-Serna P, Domínguez-Espinosa G, Díaz-Calleja R, Riande E, Alegría L, Gargallo L, Radic’ D. Macromolecules 2010; 43:5723.

[21] Sanchis MJ, Ortiz-Serna P, Carsí M, Díaz-Calleja R, Riande E, Gargallo L, Radic’ D. The Journal of Physical Chemistry 2011; 115:5730. 
[22] Roe RJ, Rigby D, Furuya H, Takehuchi H, Computational Polymer Science 1992; 2(1):32.

[23] Takeuchi H, Roe RJ, J. Chem. Phys. 1991;94:7446.

[24] Boyd R, Smith G. Polymer Dynamics and Relaxation, Cambridge University Press 2007

[25] Boyd RH Polymer 1985; 26:323.

[26] Boyd RH. Polymer 1985; 26:1123.

[27] Buerger DE, Boyd RH Macromolecules 1989; 22:2694.

[28] Buerger DE, Boyd RH. Macromolecules 1989; 22:2699.

[29] Heijboer, J. In Physics of Non-Crystalline Solids; Prins, J. A., Ed.; North-Holland, Amsterdam; 1965. p. 231.

[30] Hiroyuki S, Shogo S. Journal of Polymer Science Part A-2: Polymer Physics 1968; 6(8):1401-1418.

[31] Cowie JMG. J. Macromol. Sci., Part B: Phys. 1980; 18:569.

[32] Meier G, Fytas G, Dorfmüller T. Macromolecules 1984; 17:957.

[33] Giebel L, Meier G, Fytas G, Fischer EW. J. Polym. Sci., Part B: Polym. Phys. 1992; 30:1291.

[34] Garwe F, Schönhals A, Lockwenz H, Beiner M, Schröter K, Donth E. Macromolecules 1996; 29:247.

[35] Schröter K, Unger R, Reissig S, Garwe F, Kahle S, Beiner M, Donth E. Macromolecules 1998; 31:8966.

[36] Floudas G, Stepanek P. Macromolecules 1998; 31:6951.

[37] Beiner M, Schröter K, Hempel E, Reissig S, Donth E. Macromolecules 1999; 32:6278.

[38] Dudognon E, Bernès A, Lacabanne C. Macromolecules 2001; 34:3988.

[39] Dudognon E, Bernès A, Lacabanne C. Macromolecules 2002; 35:5927.

[40] Beiner M. Macromol. Rapid Commun. 2001; 22:869.

[41] Beiner M, Kabisch O, Reichl S, Huth HJ. Non-Cryst. Solids 2002; 307:658.

[42] Hempel E, Beiner M, Huth H, Donth, E. Thermochim. Acta 2002; 391:219.

[43] Pascui O, Beiner M, Reichert D. Macromolecules 2003; 36:3992.

[44] Beiner M, Huth H. Nat. Mater. 2003; 2:595.

[45] Hiller S, Pascui O, Kabisch O, Reichert D, Beiner M. New J. Phys. 2004; 6:1.

[46] Menissez C, Sixou B, David L, Vigier G. J. Non-Cryst. Solids 2005; 351:595.

[47] Wind M, Graf R, Renker S, Spiess H. W. Macromol. Chem. Phys. 2005;206:142. 
[48] Beiner, M. CP832, Flow Dynamics, The Second Internacional Conference on Flow Dynamics; Tokuyama, M., Maruyama, S., Eds.; American Institute of Physics: Melville, NY, 2006; p 134.

[49] Arbe A, Genix AC, Colmenero J, Richter D, Fouquet P. Soft Matter 2008;4:1792.

[50] Arbe A, Genix AC, Arrese-Igor S, Colmenero J, Richter D. Macromolecules 2010;43:3107-3119.

[51] Godard ME, Saiter JM, Journal Polymer Science:Polymer Physics 1998; 36:2865.

[52] Godard ME, Saiter JM, Journal of non Crystalline Solids 1998; 235-237:635.

[53] Grenet J, Saiter JM, Godard ME, Journal of non Crystalline Solids 2002; 307-310:232.

[54] Mark JE. Polymer Data Handbook, Oxford University Press, Inc. 1999.

[55] Carsí M, Sanchis MJ, Díaz-Calleja R, Riande E, Nugent M.J.D. Macromolecules 2012; 45:3571-3580.

[56] Cheng P, Driessen A, Tijsma E, Udipi1 K. J. of Controlled Release 2006;116(2):e92-e94.

[57] Fuoss R, Kirkwood JE. J. Am. Chem. Soc. 1941; 63:385.

[58] Vogel H. Z Phys 1921;22:645-646.

[59] Fulcher GS. J Am Ceram Soc 1925;8:339-340.

[60] Tamman G, Hesse W. Z Anorg Allg Chem 1926; 156:245-247.

[61] Doolittle AK. J Appl Phys 1951;22(12):1471.

[62] Doolittle AK. J Appl Phys 1952;23(2):236.

[63] Fujimori H, Oguni M. Solid State Commun 1995; 94:157.

[64] Schwarzl FR, Struik LCE. Adv Mol Relax Process 1967; 1:201.

[65] Diaz-Calleja R, Riande E, San Roman J. J. Polym Sci Part B: Polym Phys. 1992; 30:1239.

[66] Diaz-Calleja R, Riande E. J of NonCrystalline Solids 1994; 172-4:1037.

[67] Laredo E, Herandez MC. J. Polym Sci Part B: Polym Phys. 1997; 35:2879.

[68] Gómez-Ribelles J.L., Díaz-Calleja R., J. Macromol Sci.Phys B 1984; 23(2):855.

[69] Q. Qin, G.B. McKenna, Journal of Non-Crystalline 2006; 352:2977.

[70] Maxwell JC. Electricity and Magnetism, Clarendon, Oxford 1893.

[71] Wagner KW. Arch. Elektrotech. 1914; 2:371.

[72] Sillars RW. Inst Electr Eng 1937; 80:378; Mijovic J, Fitz BD. Novocontrol Applic Note Dielectrics 1998; 26.

[73] Mijovic J, Fitz BD. Novocontrol Applic Note Dielectrics 1998; 26. 


\section{Figure Caption}

Figure 1. Structure of 2 ethoxyethyl methacrylate (EEMA)

Figure 2. Structure of diethylene glycol dimethacrylate (DEGDMA)

Figure 3. Storage and loss Young's modulus as a function of the temperature for PEOEMA at several frequencies (0.3 [square], 1 [circle], 3 [up triangle], 10 [triangle bellow], 30 [diamond] $\mathrm{Hz})$.

Figure 4. Storage and loss Young's modulus as a function of the temperature for CEOEMA at several frequencies (0.3 [square], 1 [circle], 3 [up triangle], 10 [triangle bellow], 30 [diamond] $\mathrm{Hz}$ ).

Figure 5. Temperature dependence of the loss compliance function at several frequencies $(0.3$ [square], 1 [circle], 3 [up triangle], 10 [triangle bellow], 30 [diamond] Hz) for (a) CEOEMA and (b) PEOEMA. Inset shows the quality of the fit at one temperature for each polymer at $1 \mathrm{~Hz}$

Figure 6. Temperature dependence of the loss dielectric permittivity for PEOEMA and CEOEMA at several frequencies.

Figure 7. Arrhenius plots for the $\beta$ (blue square) and $\gamma$ (green triangle). dielectric relaxations. The temperature dependence of the mechanical $\gamma$ relaxations for PEOEMA and CEOEMA are represented for open and filled circles, respectively.

Figure 8. Plots showing the temperature dependence of $E^{\prime}$ (green curve), $E^{\prime \prime}$ (red curve), $d E^{\prime} / d T$ (purple curve) and $E_{a}$ (blue curve) for (a) PEOEMA and (b) CEOEMA at $30 \mathrm{~Hz}$. Figure 9. Mechanical loss Young's modulus E"and dielectric loss modulus $\mathrm{M}^{\prime \prime}$ as a function of temperature for (a) PEOEMA and (b) CEOEMA, at 10Hz. 
Table 1. Values of fit Fuoss-Kirkwood parameters, and $m$ and $\Delta D_{\gamma}$ of the $\gamma$ relaxation process at different frequencies

\begin{tabular}{|c|c|c|c|c|}
$f(\boldsymbol{H z})$ & $\boldsymbol{D}_{\max , \gamma}(\mathrm{MPa})$ & $\boldsymbol{m}_{\gamma} \cdot \boldsymbol{E a} / \boldsymbol{R}, \boldsymbol{K}$ & $\boldsymbol{m}_{\gamma}$ & $\Delta \boldsymbol{D}_{\gamma}(\mathrm{MPa})$ \\
\hline \multicolumn{5}{|c|}{ CEOEMA } \\
\hline 0.3 & $7.3 \cdot 10^{-6} \pm 1.3 \cdot 10^{-8}$ & $805 \pm 13$ & $0.122 \pm 0.000$ & $1.2 \cdot 10^{-4} \pm 3.4 \cdot 10^{-7}$ \\
1 & $7.6 \cdot 10^{-6} \pm 1.1 \cdot 10^{-8}$ & $884 \pm 14$ & $0.134 \pm 0.000$ & $1.1 \cdot 10^{-4} \pm 3.0 \cdot 10^{-7}$ \\
3 & $7.5 \cdot 10^{-6} \pm 1.3 \cdot 10^{-8}$ & $934 \pm 13$ & $0.141 \pm 0.000$ & $1.1 \cdot 10^{-4} \pm 7.3 \cdot 10^{-8}$ \\
10 & $7.8 \cdot 10^{-6} \pm 8.2 \cdot 10^{-9}$ & $916 \pm 9$ & $0.139 \pm 0.001$ & $1.1 \cdot 10^{-4} \pm-5.0 \cdot 10^{-7}$ \\
30 & $8.2 \cdot 10^{-6} \pm 1.3 \cdot 10^{-8}$ & $869 \pm 12$ & $0.132 \pm 0.000$ & $1.2 \cdot 10^{-4} \pm 1.5 \cdot 10^{-8}$ \\
\hline & \multicolumn{3}{|c|}{ PEOEMA } \\
\hline 3 & $1.2 \cdot 10^{-5} \pm 1.6 \cdot 10^{-8}$ & $696 \pm 7$ & $0.104 \pm 0.004$ & $2.3 \cdot 10^{-4} \pm 8.1 \cdot 10^{-6}$ \\
10 & $1.3 \cdot 10^{-5} \pm 1.4 \cdot 10^{-8}$ & $661 \pm 5$ & $0.099 \pm 0.004$ & $2.6 \cdot 10^{-4} \pm-9.8 \cdot 10^{-6}$ \\
30 & $1.4 \cdot 10^{-5} \pm 2.0 \cdot 10^{-8}$ & $609 \pm 5$ & $0.091 \pm 0.003$ & $3.1 \cdot 10^{-4} \pm 1.1 \cdot 10^{-5}$ \\
\hline
\end{tabular}




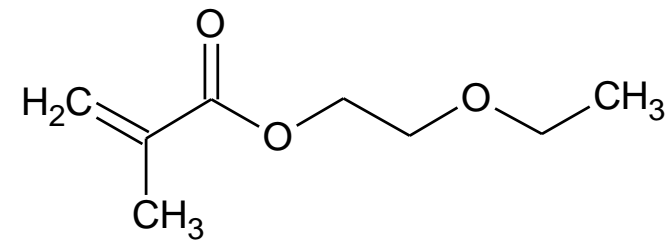

Figure 1. 


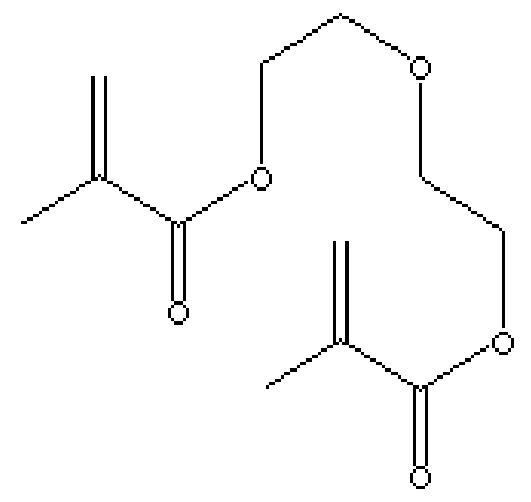

Figure 2. 

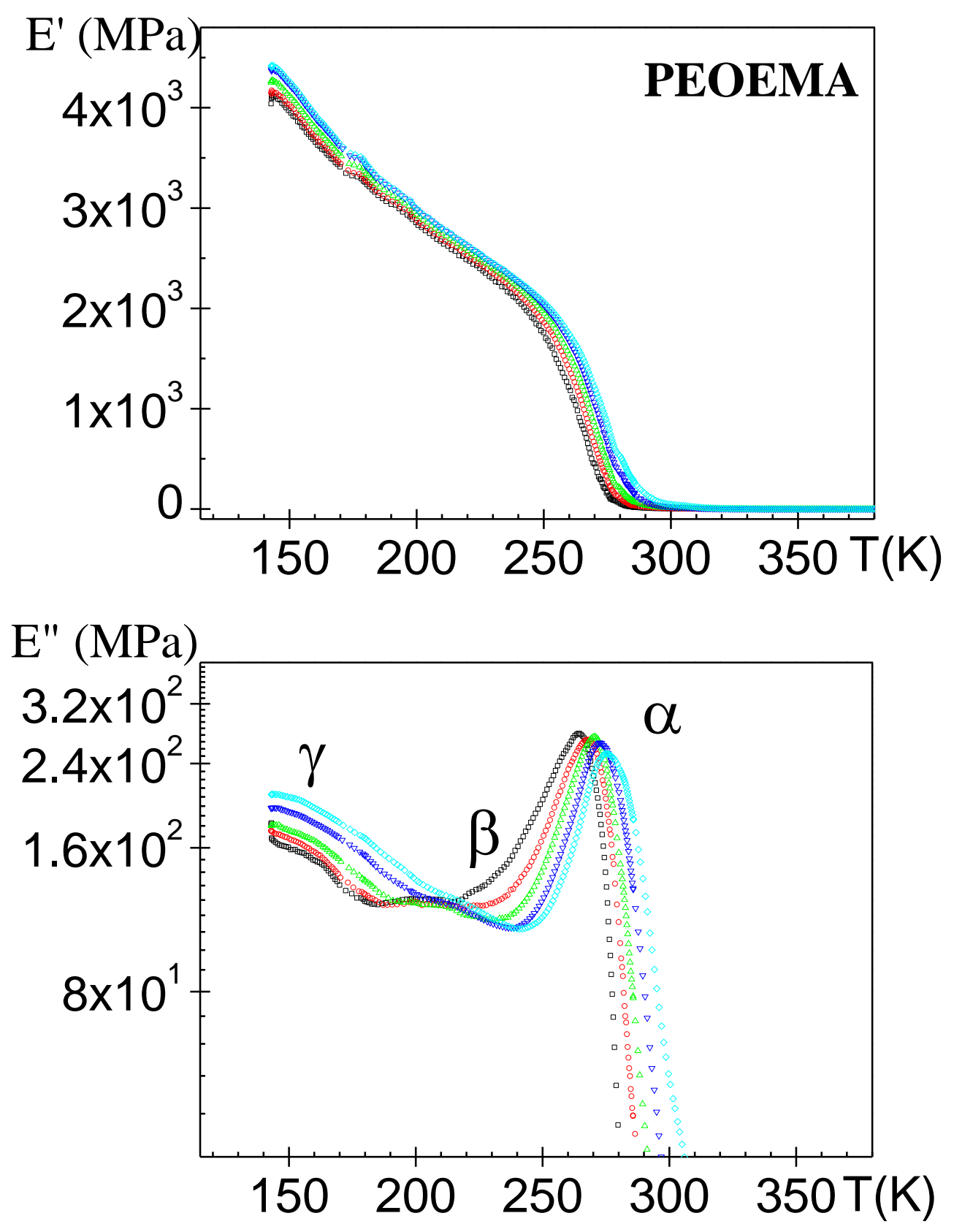

Figure 3. 

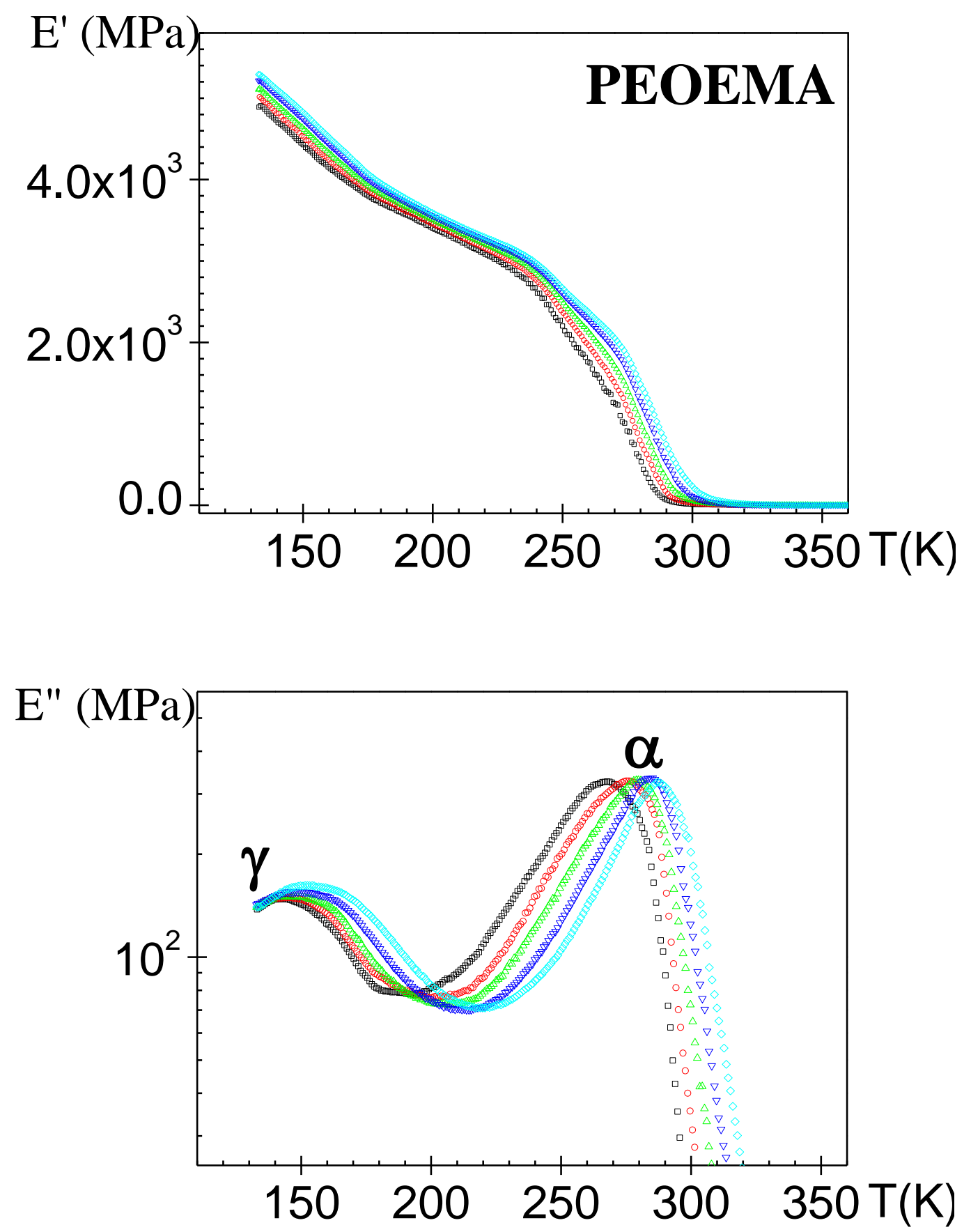

Figure 4. 

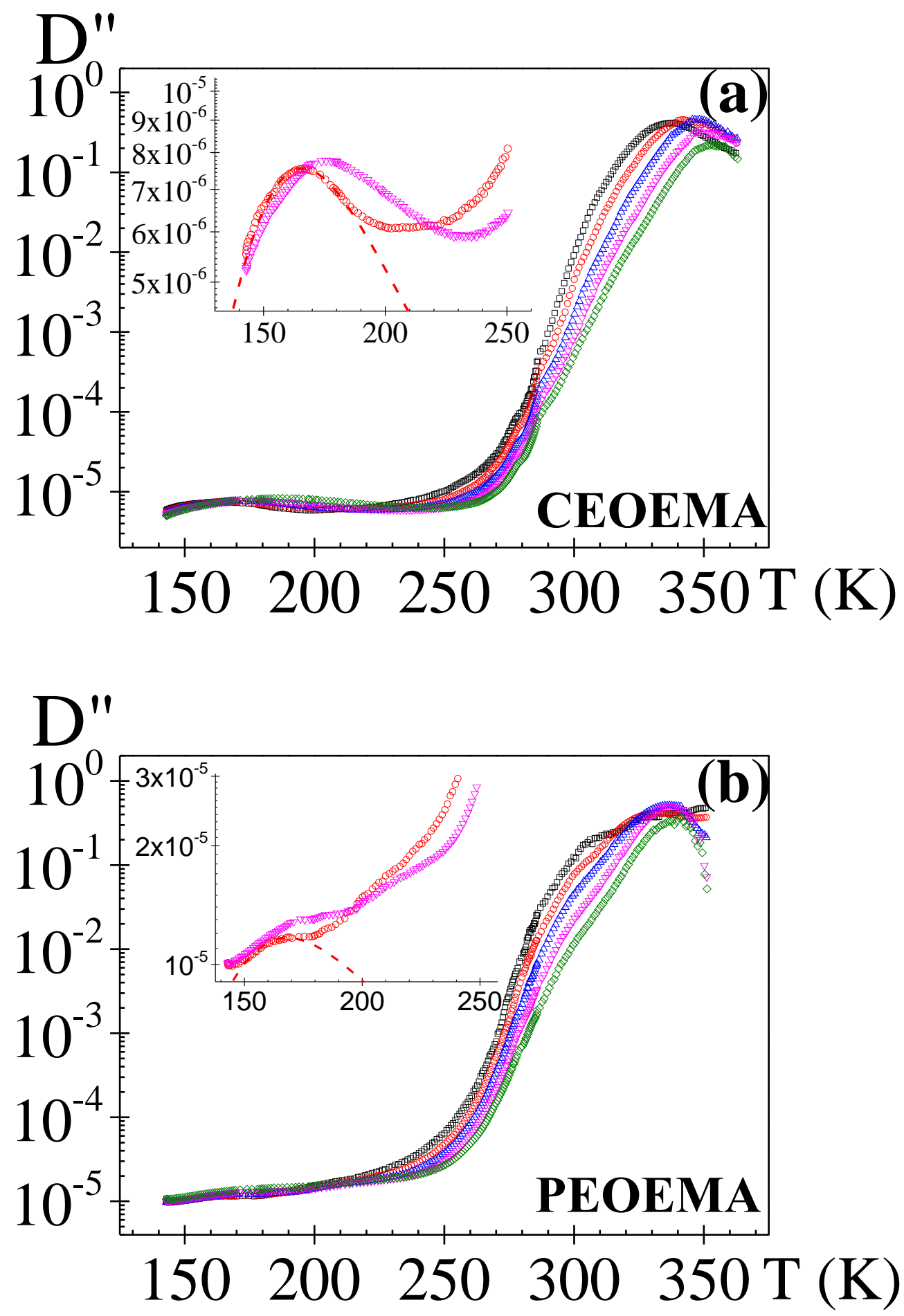

Figure 5. 


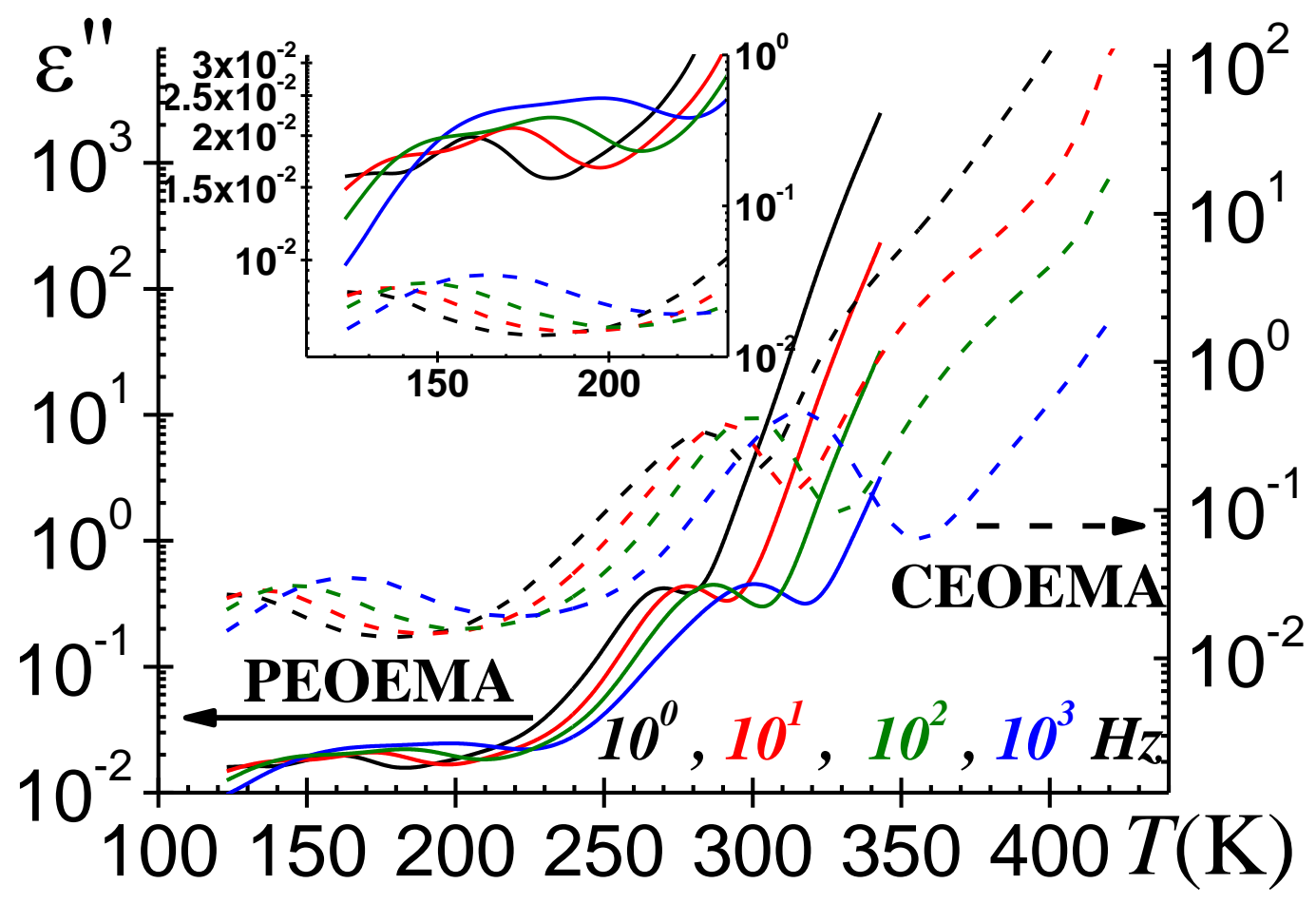

Figure 6. 


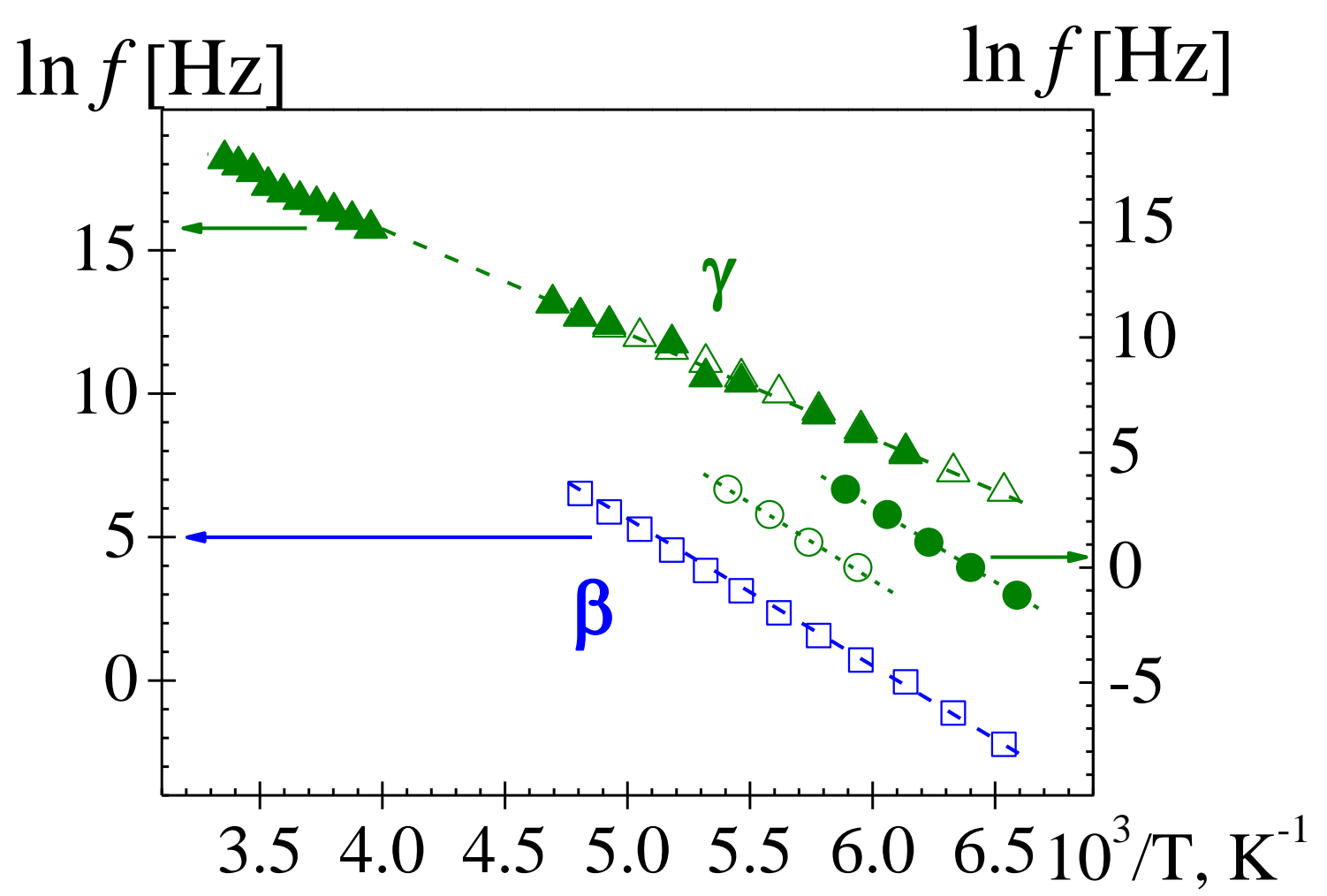

Figure 7. 

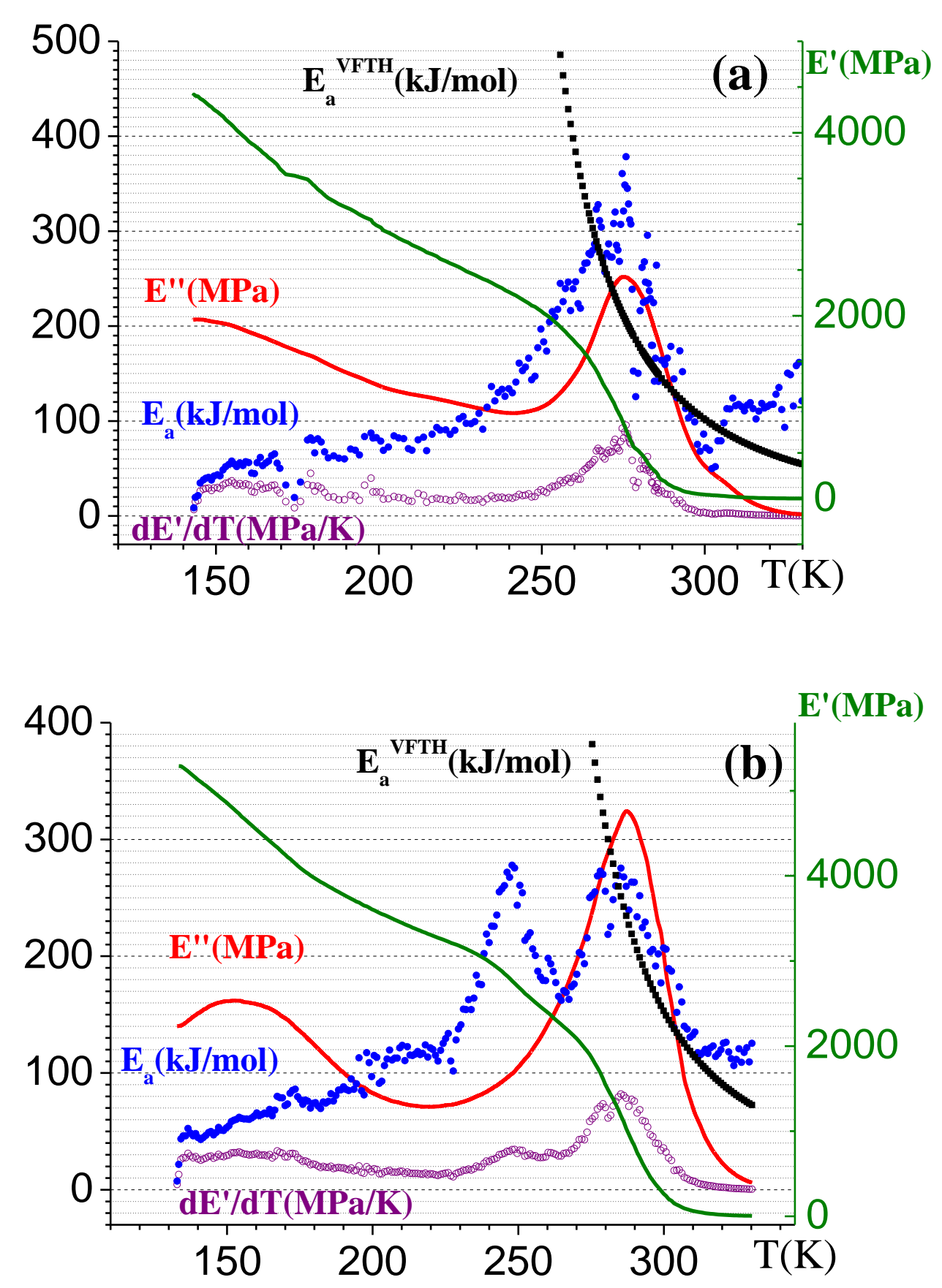

Figure 8. 

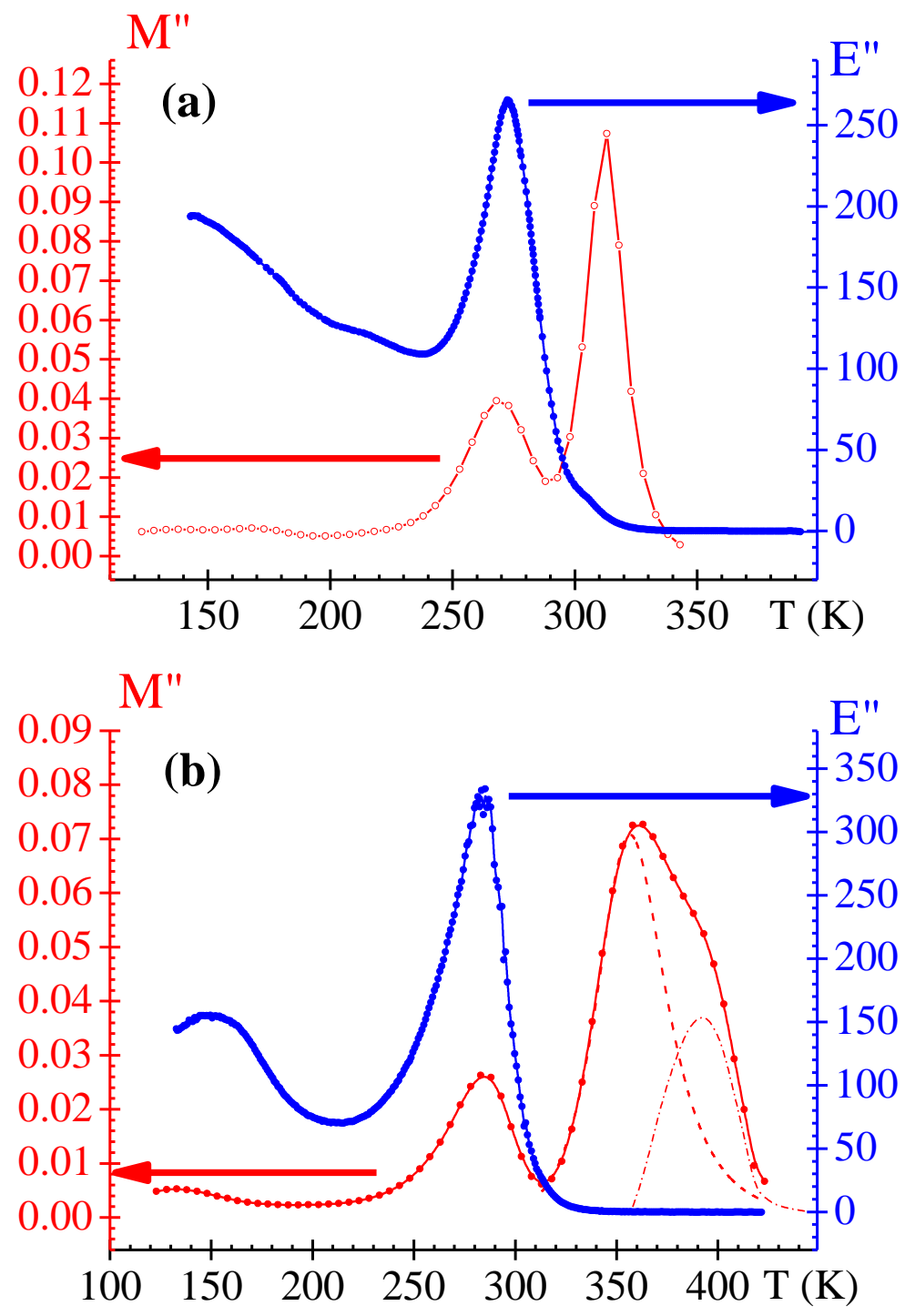

Figure 9. 
